ARTICLE

\title{
Compound jetting from bubble bursting at an air-oil-water interface
}

\author{
Bingqiang Ji (ib) ${ }^{1,3}$, Zhengyu Yang ${ }^{1,3} \&$ Jie Feng (i) ${ }^{1,2 凶}$
}

Bursting of bubbles at a liquid surface is ubiquitous in a wide range of physical, biological, and geological phenomena, as a key source of aerosol droplets for mass transport across the interface. However, how a structurally complex interface, widely present in nature, mediates the bursting process remains largely unknown. Here, we document the bubble-bursting jet dynamics at an oil-covered aqueous surface, which typifies the sea surface microlayer as well as an oil spill on the ocean. The jet tip radius and velocity are altered with even a thin oil layer, and oily aerosol droplets are produced. We provide evidence that the coupling of oil spreading and cavity collapse dynamics results in a multi-phase jet and the follow-up droplet size change. The oil spreading influences the effective viscous damping, and scaling laws are proposed to quantify the jetting dynamics. Our study not only advances the fundamental understanding of bubble bursting dynamics, but also may shed light on the airborne transmission of organic matters in nature related to aerosol production.

\footnotetext{
${ }^{1}$ Department of Mechanical Science and Engineering, University of Illinois at Urbana-Champaign, Urbana, IL 61801, USA. ${ }^{2}$ Materials Research Laboratory, University of Illinois at Urbana-Champaign, Urbana, IL 61801, USA. ${ }^{3}$ These authors contributed equally: Bingqiang Ji, Zhengyu Yang.

凶email: jiefeng@illinois.edu
} 
B ubbles are widely observed at liquid surfaces, as they are easily formed by natural processes, such as wave breaking, raindrop impacts, and even changes of the solubility of air in water due to weather and seasons ${ }^{1}$. These surface bubbles eventually burst once the liquid film that forms the bubble cap drains to rupture, generating film droplets by cap disintegration, as well as jet droplets by fragmentation of the upwardly directed liquid jet formed at the end of cavity collapse ${ }^{2}$. These small droplets can remain suspended in the air, and therefore play a key role in mediating the mass transport across the interface in a wide range of physical, geological, and biological phenomena, including the flavor release from sparkling drinks ${ }^{3}$, sea spray aerosol generation for climate ${ }^{4-8}$, and even facilitation of vegetative reproduction over the ocean ${ }^{9}$.

Recently, bubbles have also been recognized as a major public health concern, regarding the aerosolization of contaminated water with harmful substances for airborne transmission of pollutants and infectious diseases ${ }^{10,11}$. In this context, the interplay between bubbles and a contaminated interface is a crucial factor for the aerosol droplet generation. In essence, rising bubbles scavenge surface-active organic material from the water column at their interfaces, and this process facilitates the formation of an organic-enriched air-liquid interface ${ }^{12,13}$. Examples include the sea-surface microlayer on the ocean, or the microorganismenriched interface in the reaction tanks of municipal waste-water treatment plants ${ }^{14,15}$. Although some recent studies show that bubble bursting can disperse the surface organic layer as submicrometer droplets into water ${ }^{16,17}$ or eject organic matter into the atmosphere through film droplets ${ }^{18,19}$, the capability of such a chemically complex interface to manipulate the bubble physics, particularly the jetting, remains largely unknown. In particular, jet droplets play an important role in the mass flux of bubble bursting aerosols ${ }^{4}$. For instance, it has been found that jet droplets produce up to $43 \%$ of total submicrometer sea spray aerosol number concentrations ${ }^{15}$.

Here, we investigate the bubble bursting dynamics at an oilcovered aqueous surface by high-speed imaging, to characterize the previously unknown interplay between bubbles and a structurally complex interface with an insoluble viscous oil layer. We show that a compound multiphase jet is formed, resulting from the spreading of the oil layer on the air-water surface of the bubble cavity in a complete-wetting state. We found that even highly viscous oil can be ejected into the atmosphere with the compound jetting, where the oil layer viscosity is far beyond the maximum viscous limit for a bubble bursting jet to produce droplets in a single liquid. By modeling the extra viscous dissipation regarding the oil film spreading along the cavity surface, we propose scaling laws that quantify the contribution of the oil viscosity and oil layer thickness to the change in the radius and velocity of the jet tip. Our results provide potential guidance for the prediction of organic-enriched aerosol produced by bubble bursting at a structurally complex interface.

\section{Results}

High-speed imaging of bubble bursting. The bubble bursting dynamics at an oil-covered aqueous surface is observed by two synchronized high-speed cameras from side views above and below the liquid surface (Fig. 1a). No surface-active material is added in any liquid. The gas bubble approaches the oil-covered aqueous surface after being released. Then the bubble rests at the air-oil-water interface with a cap consisting of water and oil films after its initial oscillation has died down (Fig. 1b), and finally bursts. The bubble shape at rest is almost unchanged in the pure water and different oily cases. We fixed the equivalent radius of the bubble as $R=1.67 \mathrm{~mm}$ and used $5-1000 \mathrm{cSt}$ silicone oils in our experiments (Table 1). When the oil layer thickness $h$ is larger than a critical thickness $h_{\mathrm{c}}(\approx 1.2 R)$, the water film on the bubble cap ruptures first, and subsequently the whole bubble enters the oil layer. In such a regime, no jet droplets are emitted by bubble bursting at the viscous oil layer in the experiments (Supplementary Fig. 1 and Supplementary Movies 1-3). While for $h \leq h_{\mathrm{c}}$, the oil and water films on the bubble cap rupture simultaneously, and the subsequent cavity collapse results in a compound multiphase jet that ejected droplets. Therefore, all experiments were performed with $h \leq h_{\mathrm{c}}$ (Fig. 1c).

Oily jetting. In our experimental system, after the simultaneous rupture of the oil and water films on the bubble cap, the cavity collapses, with capillary waves propagating downwards and finally focusing at the cavity nadir. Then the curvature of the interface at the bubble nadir reverses, generating an upward Worthington jet ${ }^{20}$ that finally breaks up due to Rayleigh-Plateau instability and disperses myriads of droplets, known as jet droplets, into air. Surprisingly, unlike the bubble bursting jet from a clean water surface ${ }^{3,21,22}$, a long and thin liquid thread appears during the jet pinch-off from an oil-covered aqueous surface (Fig. 2b-d). Such a feature is a typical characteristic of highly viscous fluid pinch-off ${ }^{23,24}$, which indicates the formation of an oily jet. In addition, we observed dumbbell-shaped jet droplets produced by such a compound jet (Supplementary Fig. 2 and Supplementary Movie 4), which is another distinct feature compared with bubble bursting at a clean water surface. These droplets remain uncoalesced until they fall onto the oil layer with a much longer life time compared with pure water droplets. We believe the oil coating delays the droplet coalescence, as a further evidence for the compound droplets from the oily jetting. In addition, by dispersing tracer microparticles into the oil layer and imaging the particles from the dried top jet droplet collected on a glass slide, we directly evidenced the presence of oil in the jet droplets (Supplementary Fig. 3), and the top jet droplet could contain an oil volume fraction of up to $83 \%$ (see SI for the calculation).

It has been well established that the jet formation after the cavity collapse controls the follow-up droplet generation, including the jet droplet size and velocity, as shown in prior work for bubble bursting at a single liquid surface ${ }^{25,26}$. In particular, the jet tip radius and velocity show similar trends as the top jet droplet radius and velocity ${ }^{26}$. Therefore, we mainly focus on characterizing the jet dynamics in the current work, as a first step to inform the interplay between bubbles and a compound interface. The radius, $r_{j}$, and velocity, $v_{j}$, of the jet tip are measured when the tip crosses the horizontal level of undisturbed air-oil interface (Fig. 2a). Remarkably, we observe that the oily jet is thinner and travels faster, i.e., $r_{\mathrm{j}}$ decreases and $v_{\mathrm{j}}$ increases with increasing oil viscosity $\left(\mu_{\mathrm{o}}\right)$ and oil layer thickness (h) (Fig. 2e, f), and eventually smaller oily jet droplets are ejected with a faster speed compared with the case of an air-water surface (Fig. $2 \mathrm{a}-\mathrm{d}$ ). The radius and velocity of the top jet droplet at the moment of detachment are also measured, as shown in Supplementary Fig. 4. Meanwhile, both the jetting height and jet droplet number increase with $\mu_{\mathrm{o}}$ and $h$ (Supplementary Movie 5).

Previous studies show that the jet dynamics of bubble bursting at a clean liquid surface is mainly controlled by two dimensionless numbers, e.g., the Bond number Bo $=\rho g R^{2} / \gamma$ and Ohnesorge number

$$
\mathrm{Oh}=\frac{\mu}{\sqrt{\rho \gamma R}},
$$

where $\rho, \mu, \gamma$ indicate liquid density, dynamic viscosity, and interfacial tension, respectively, and $g$ is the gravitational 

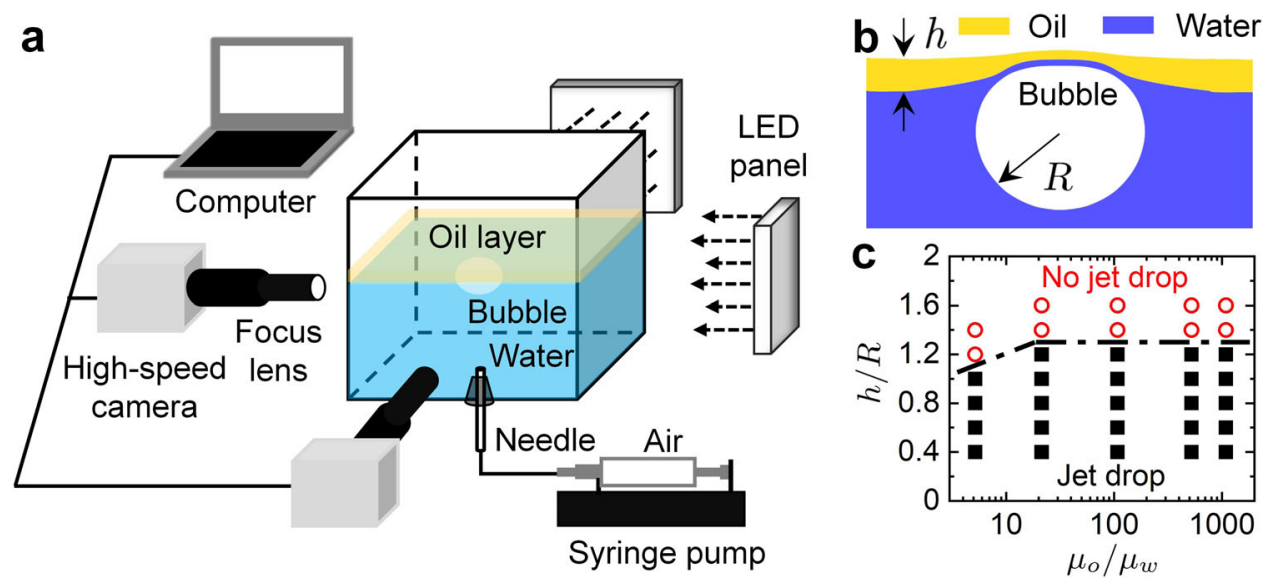

Fig. 1 Experimental setup, sketch of a still bubble at an oil-covered aqueous surface and regime map of bubble bursting jets. a Schematic of the experimental apparatus. The air bubble with an equivalent radius of $R=1.67 \mathrm{~mm}$ is generated by a needle at the bottom of the container with a syringe pump, and it rises to the water surface covered by an oil layer with a thickness $h$. The bubble rests at the oil-covered aqueous surface, and then bursts, which is recorded by two computer-controlled synchronized high-speed cameras with LED illumination. $\mathbf{b}$ Close-up sketch of an air bubble with radius $R$ resting at an aqueous surface covered by an oil layer with a thickness $h$, which typifies an organic-enriched, structurally complex interface. c Regime map of bubble bursting jets at an oil-covered aqueous surface regarding the oil viscosity $\mu_{\mathrm{o}}$ and the oil layer thickness $h$, showing the boundary between no jet droplet and jet droplet of the bubble bursting. Jet droplets only occur when the oil layer thickness is smaller than a critical thickness $h_{c}$, where the oil and water layers rupture simultaneously and oily jet droplets are ejected into air. When $h>h_{c}$, the water film ruptures first and then the whole bubble enters the oil layer, and the subsequent bubble bursting jet does not eject any oily aerosol droplets (Supplementary Fig. 1 and Supplementary Movies 1-3).

Table 1 Physical properties of the liquids used in the experiments.

\begin{tabular}{|c|c|c|c|c|c|}
\hline Liquids & $\rho\left(\mathrm{kg} / \mathrm{m}^{3}\right)$ & $\mu(\mathrm{mPa} \cdot \mathrm{s})$ & $\gamma_{w a}(\mathrm{mN} / \mathrm{m})$ & $\gamma_{o a}(\mathrm{mN} / \mathrm{m})$ & $\gamma_{\text {ow }}(\mathrm{mN} / \mathrm{m})$ \\
\hline DI water & 998 & 0.89 & $71.6 \pm 1.0$ & $\mathrm{~N} / \mathrm{A}$ & $\mathrm{N} / \mathrm{A}$ \\
\hline 5 cSt silicone oil & 913 & 4.6 & $\mathrm{~N} / \mathrm{A}$ & $18.7 \pm 0.3$ & $38.1 \pm 0.4$ \\
\hline 20 cSt silicone oil & 950 & 19 & $\mathrm{~N} / \mathrm{A}$ & $19.4 \pm 0.7$ & $40.9 \pm 0.5$ \\
\hline 500 cSt silicone oil & 970 & 465 & $\mathrm{~N} / \mathrm{A}$ & $19.9 \pm 0.1$ & $38.7 \pm 0.2$ \\
\hline 1000 cSt silicone oil & 970 & 970 & $\mathrm{~N} / \mathrm{A}$ & $21.7 \pm 0.2$ & $40.8 \pm 1.0$ \\
\hline
\end{tabular}

acceleration $^{21,25,27}$. Under conditions in which Bo (effect of gravity relative to surface tension) exceeds 3 , no jet droplets are produced because of the influence of gravity on the cavity shape. Similarly, when Oh (effect of viscosity to inertial and surface tension) exceeds $0.03-0.04$, jet droplets are not produced since viscous stresses suppress jetting by damping out the inertial capillary waves driving the motion ${ }^{28,29}$.

Using the silicone oil properties in our current experiments (Table 1), Bo $=1.2-1.4$ and $\mathrm{Oh}=0.03-5$, no jet droplet is expected by bubble bursting at the surface of a bulk silicone oil ${ }^{28}$. However, the ejection of the oily jet droplet is observed in our experiment, even if the oil layer viscosity is high. Therefore, for a compound interface with a viscous oil layer on top of the water surface, bubble bursting jet can actually eject droplets containing the very viscous oil component as shown by our study. This feature is not predicted by the maximum viscous limit, considering $\mathrm{Oh}$, for jet droplets to form at a clean bulk liquid surface in prior work. This indicates that for an organic-enriched interface, such as the sea-surface microlayer, the highly viscous surface organic matter may be ejected into the atmosphere through the break-up of a bubble bursting jet, raising the question of how the oils at the aqueous surface transport to the jet and jet droplets produced by a clean gas bubble.

Oil spreading on cavity surface. Here we show that the formation of oily jets results from the spreading of oils along the surface of the collapsing cavity. For the bubble dynamics at an air-oil-water interface, it is important to consider physicochemical interaction between oil and water molecules, as highlighted by our previous work about nanoemulsions produced by bubble bursting ${ }^{16,30}$. The bubble cavity surface below the oil-water interface before bubble bursting is initially oil-free as shown in Fig. 1b. Given the positive spreading coefficient $\Delta \gamma=$ $\gamma_{\mathrm{wa}}-\gamma_{\mathrm{oa}}-\gamma_{\mathrm{ow}}>0$ (Table 1) in the complete-wetting state between silicone oil and water, the oil spontaneously spreads along the cavity surface towards the bubble nadir once the cap ruptures. In addition, it has been shown by previous studies ${ }^{31,32}$ that the spreading of a silicone oil droplet on an aqueous surface exhibits two distinct spreading fronts with non-uniform thicknesses, i.e., a faster microscopic front connecting to a slower macroscopic bulk edge. From the high-speed imaging as shown in Fig. 3 and Supplementary Fig. 5, a macroscopic oil edge propagating along the cavity surface is observed. In addition, the propagation of the macroscopic spreading edge is slower with increasing oil viscosity (Fig. 3b, c) but basically unaffected by the oil layer thickness (Fig. 3c, d). For instance, the macroscopic spreading edge of $100 \mathrm{cSt}$ oil arrives at the cavity nadir at the onset of jet formation ( $t=2.8 \mathrm{~ms}$ in Fig. 3b), and that of $1000 \mathrm{cSt}$ oil is still far away from the cavity nadir $(t=3.6 \mathrm{~ms}$ in Fig. $3 \mathrm{c}, \mathrm{d})$ when the nadir curvature reverses.

While we cannot directly visualize the microscopic front ahead of the macroscopic oil edge since the adjacent oil-film thickness is 


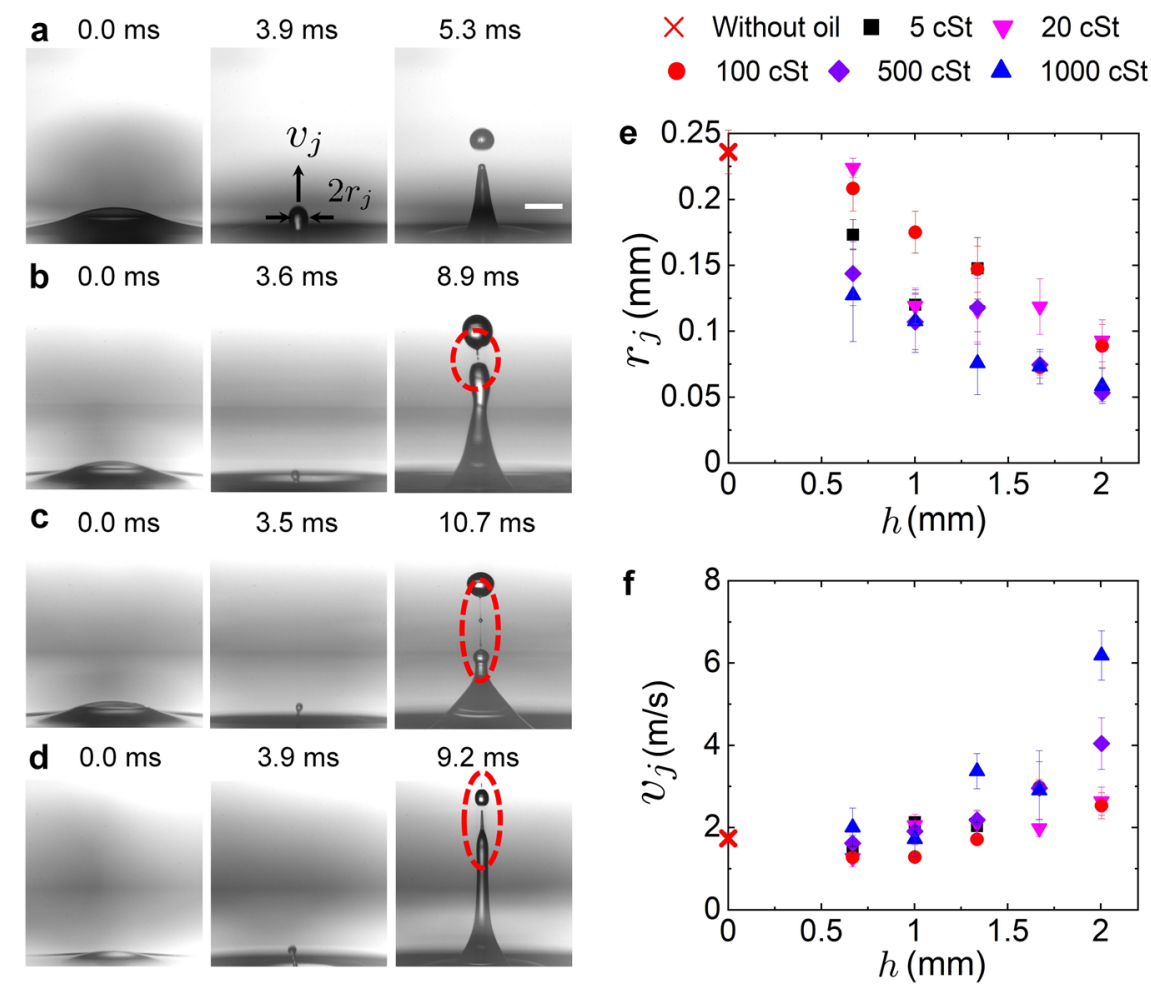

Fig. 2 Snapshots of bubble bursting jetting and the measured jet tip radius and velocity. Side-view snapshots of jet formation during bubble bursting at the aqueous surface (a) without an oil layer, and covered by a layer of (b) $5 \mathrm{cSt}$ silicone oil, $h / R=0.6$, (c) $500 \mathrm{cSt}$ silicone oil, $h / R=0.6$, and (d) $500 \mathrm{cSt}$ silicone oil, $h / R=1.2 . R=1.67 \mathrm{~mm}$ in the experiments. Scale bar $=1 \mathrm{~mm} . t=0$ represents the beginning of bubble bursting when a hole nucleates in the bubble cap. An oily jet is generated (column 2 of a-d) following the cavity collapse, which becomes smaller but faster with the increasing viscosity and thickness of the oil layer. A thin oily thread is observed in all oily cases (highlighted in column 3 of $\mathbf{b}$-d). Dependencies of (e) the jet tip radius $r_{j}$ and (f) jet tip velocity $v_{\mathrm{j}}$ on the oil layer thickness $h$ for different oil viscosities. $r_{\mathrm{j}}$ and $v_{\mathrm{j}}$ are measured when the jet tip crosses the undisturbed air-oil interface. Error bars are calculated as the standard deviations of data of at least 10 runs.

a $\quad 0 \mathrm{~ms}$

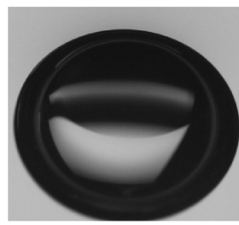

b $\quad 0 \mathrm{~ms}$
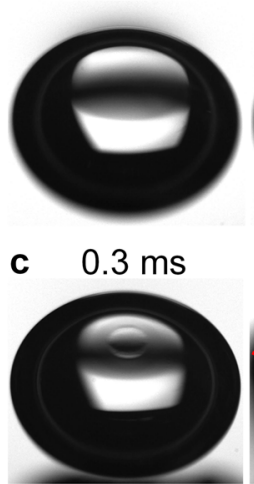

$0.2 \mathrm{~ms}$

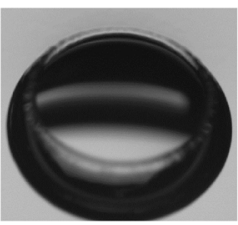

$0.2 \mathrm{~ms}$

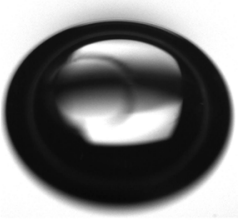

$1.5 \mathrm{~ms}$

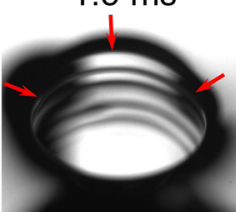

$0.9 \mathrm{~ms}$

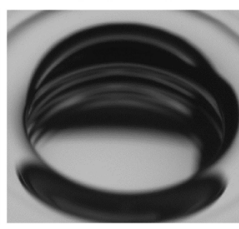

$1.3 \mathrm{~ms}$

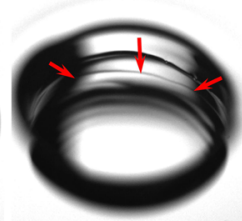

$3.6 \mathrm{~ms}$

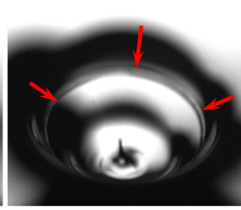

$1.8 \mathrm{~ms}$

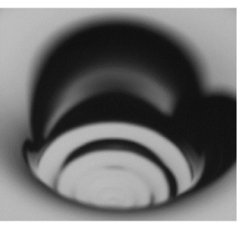

$2.1 \mathrm{~ms}$
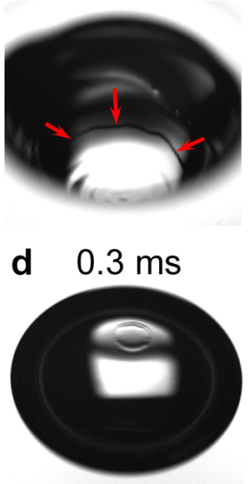

$2.7 \mathrm{~ms}$

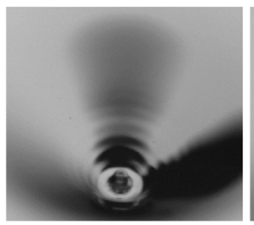

$2.8 \mathrm{~ms}$

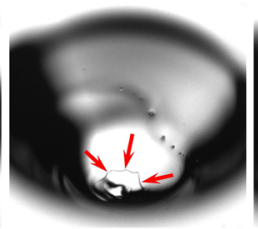

$2.0 \mathrm{~ms}$

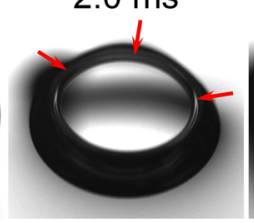

$4.7 \mathrm{~ms}$

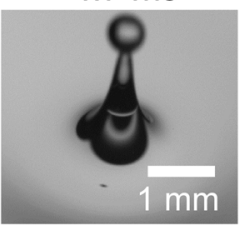

$4.0 \mathrm{~ms}$

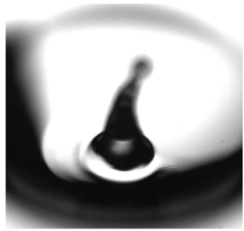

$3.6 \mathrm{~ms}$

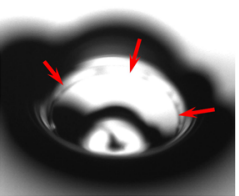

Fig. 3 Top-view snapshots of oil spreading on the cavity surface during bubble bursting at the aqueous surface. The water surfaces shown here are (a) clean, and covered by a layer of (b) $100 \mathrm{cSt}$ silicone oil, $h / R=0.8,(\mathbf{c}) 1000 \mathrm{cSt}$ silicone oil, $h / R=0.8$, and (d) $1000 \mathrm{cSt}$ silicone oil, $h / R=1.2$. Scale bar $=1 \mathrm{~mm}$. Comparing with the pure water case (a), a macroscopic oil edge (marked by red arrows) along the cavity after the cap rupture at an oilcovered aqueous surface is clearly observed, which is generated by oil spreading in the complete-wetting state (b-d). The macroscopic oil edges can be distinguished since they typically show asymmetric and irregular morphology, while the capillary waves from cavity collapse show axisymmetric and smooth patterns. Additional evidence is shown in Supplementary Fig. 5 with tracer particles to assist visualization of the macroscopic oil edges. 

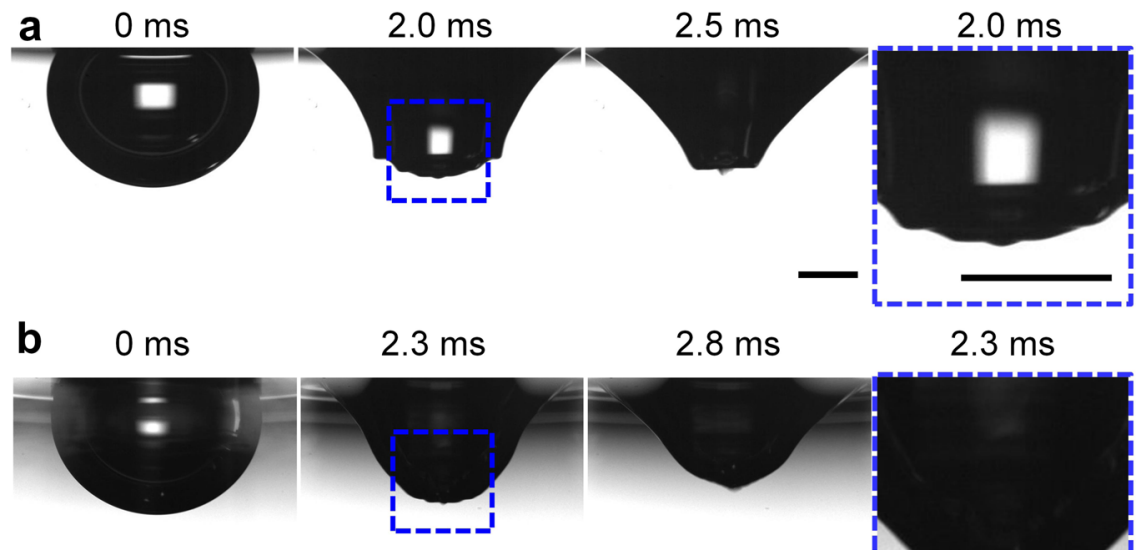

\section{$2.3 \mathrm{~ms}$}

C $\quad 0 \mathrm{~ms}$

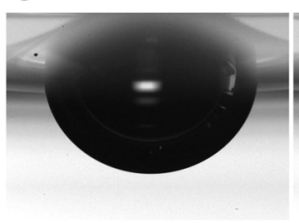

d $\quad 0 \mathrm{~ms}$

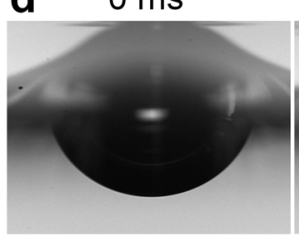

$2.3 \mathrm{~ms}$

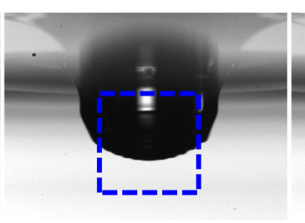

$3.4 \mathrm{~ms}$

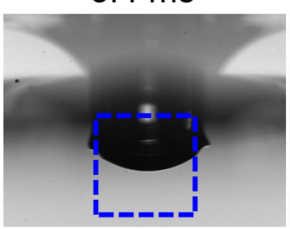

$2.8 \mathrm{~ms}$

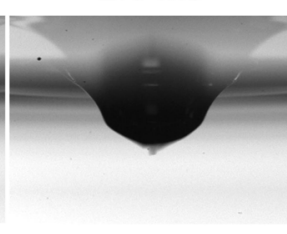

$3.9 \mathrm{~ms}$

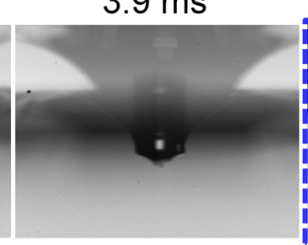

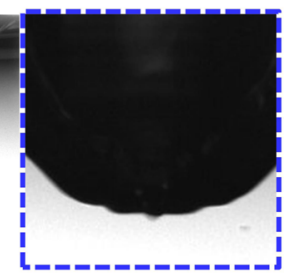

$2.3 \mathrm{~ms}$

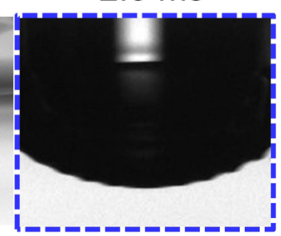

$3.4 \mathrm{~ms}$

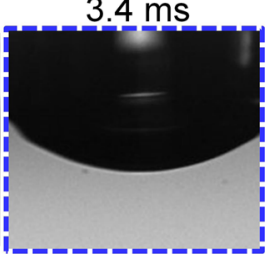

Fig. 4 Side-view snapshots of cavity collapse during bubble bursting. The water surfaces shown here are (a) clean, or covered by a layer of (b) 100 cSt silicone oil, $h / R=0.8$, (c) 1000 cSt silicone oil, $h / R=0.8$, and (d) 1000 cSt silicone oil, $h / R=1.2$. The equivalent bubble radius $R=1.67$ mm in the experiments. Scale bars represent $1 \mathrm{~mm}$. The capillary waves during cavity collapse are progressively damped in the oily cases compared with those in the pure water case (column 4 as the zoom-in images of the blue square zones in column 2), which results in a sharper tip at the cavity nadir in the oily cases (column 3), indicating the additional damping effect from the oil layer.

at the order of $O(1 \mathrm{~nm})^{33}$, the surface-tension-driven spreading speed of the microscopic oil front can be estimated by balancing the net driving force and the viscous force that developed at the boundary layer of the supporting fluid. Then the time for the microscopic oil front reaching the bubble cavity nadir can be estimated as $31,32,34$

$$
t_{\mathrm{s}} \sim\left(\frac{\mu_{\mathrm{w}} \rho_{\mathrm{w}} R^{4}}{\Delta \gamma^{2}}\right)^{1 / 3}
$$

while the time of cavity collapse can be written as the capillary time $e^{25,35}$ calculated based on the effective surface tension of the air-oil-water interface $\gamma_{\mathrm{e}}=\gamma_{\mathrm{oa}}+\gamma_{\mathrm{ow}}{ }^{36}$,

$$
t_{\mathrm{c}} \sim\left(\frac{\rho_{\mathrm{w}} R^{3}}{\gamma_{\mathrm{e}}}\right)^{1 / 2} .
$$

In our experiments, we find $t_{\mathrm{s}} \approx t_{\mathrm{c}} \approx O(1 \mathrm{~ms})$. The comparable time scales indicate that the microscopic oil front is able to reach the base of the cavity and the compound jet can form, confirming our observations. In addition, we find that a more viscous substrate liquid than water weakened the effect of the oil layer on the jet dynamics (Supplementary Fig. 6), presumably due to the slower spreading of the oil film (larger $t_{\mathrm{s}}$ ) on the cavity surface, showing the important role of the oil spreading dynamics.
Cavity collapse and capillary wave propagation. We further investigate how the oil spreading at the cavity surface modifies the jet dynamics. It has been stated that the capillary wave propagation during cavity collapse plays an important role in the jet dynamics produced by bubble bursting. During cavity collapse, the rim retraction after the bubble cap rupture excites a series of capillary waves propagating along the cavity surface (Fig. 4). The capillary waves with relatively smaller wavelengths travel faster and are more rapidly attenuated by viscosity ${ }^{25,27}$. The dominant capillary wave selected by viscous attenuation, i.e., the capillary wave with the shortest wavelength not attenuated by viscosity during cavity collapse, can successfully propagate to the bubble nadir and makes the cavity collapse to a truncated cone, which triggers the ejection of a jet ${ }^{25}$.

Considering the essential role of capillary wave dynamics, we measured the selected dominant wave speed, $v_{\mathrm{d}}$, as the propagation speed when the last wave crest crosses the position with an angle of $\pi / 4$ corresponding to the vertical cavity axis following the previous study ${ }^{25}$. We found that $v_{\mathrm{d}} / v_{\mathrm{c}}=7.2 \pm 1.3$ which is almost unaffected by the oil layer (Fig. 5), with the inertial capillary velocity $v_{\mathrm{c}}$ calculated based on $\gamma_{\mathrm{e}}$ as

$$
v_{\mathrm{c}}=\left(\frac{\gamma_{\mathrm{e}}}{\rho_{\mathrm{w}} R}\right)^{1 / 2} \text {. }
$$

This velocity ratio is consistent with that in previous numerical study on bubble bursting jetting at a clean liquid surface as 


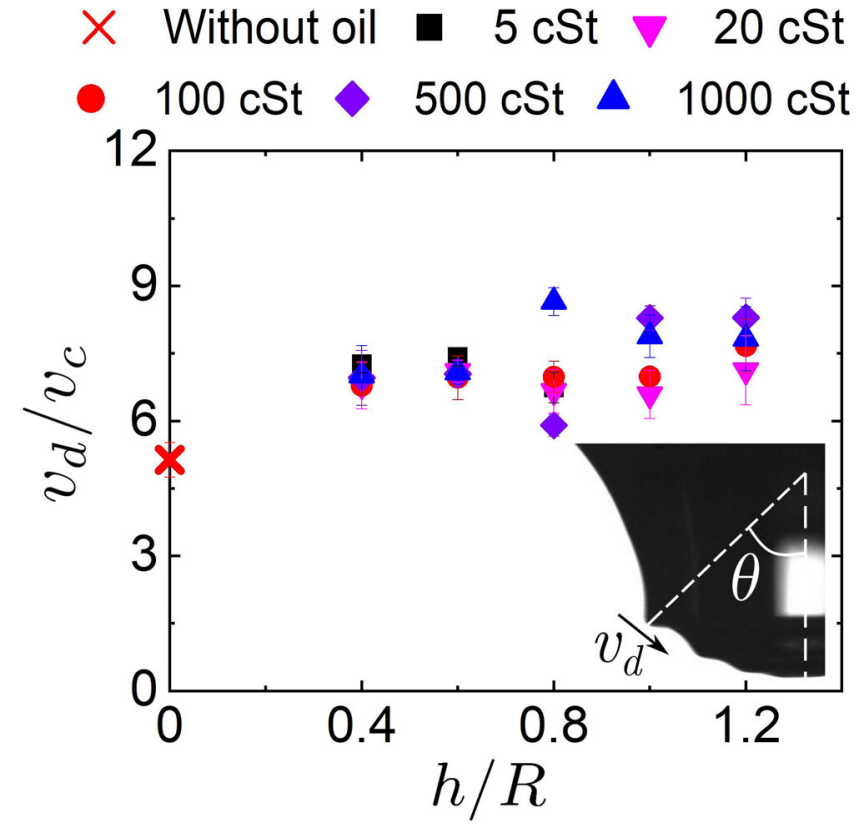

Fig. 5 Capillary wave velocity. Dependence of dimensionless velocity $v_{d} / v_{c}$ of the selected dominant capillary wave on the dimensionless oil layer thickness $h / R$ during bubble bursting at the oil-covered aqueous surface, where $v_{c}=\left(\frac{y_{e}}{\rho_{\mathrm{w}}} R\right)^{1 / 2}$ is the inertial capillary velocity. Inset shows that $v_{\mathrm{d}}$ is measured when the selected dominant wave goes through the position with an angle of $\theta=\pi / 4$ corresponding to the vertical cavity axis. Error bars are calculated as the standard deviations of data of at least 10 runs.

$v_{\mathrm{d}} / v_{\mathrm{c}} \approx 5$, which is independent of $\mathrm{Oh}^{37}$. The wavelength of the selected dominant wave, $\lambda_{\mathrm{d}}$, which was suggested to be estimated as the minimum radii of curvature of the capillary waves, was found to increase with $\mathrm{Oh}$ due to the larger viscous attenuation $^{25,37}$. An increasing $\lambda_{\mathrm{d}}$ can shorten the lower base of the truncated cone-shaped cavity, and hence decreases the jet radius and increases the jet velocity when $\mathrm{Oh}<0.03$ for bubble bursting at a clean liquid surface ${ }^{25,26,37}$. Though we could not precisely measure the radii of curvature of the capillary waves due to the spatial resolution limit of the experimental images, a smaller radius at the lower base of the truncated cone-shaped cavity is observed at the moment of jet formation when increasing the oil viscosity and layer thickness (column 3 in Fig. 4), suggesting a larger $\lambda_{\mathrm{d}}$. Meanwhile, we found that while the bubble cavity shapes remain similar, the amplitudes of the capillary waves are noticeably damped by the presence of oil (Fig. 4), and the damping effect increases with $\mu_{\mathrm{o}}$ and $h$. With increasing oil viscosity and layer thickness, more capillary waves with small wavelengths are completely damped (columns 2, 4 in Fig. 4) and the cavity profile becomes smoother. The similar trend has also been reported by previous experimental studies, which argued that with increasing $\mathrm{Oh}$, the capillary waves are progressively damped, resulting in a smaller and faster jet t $^{3,21,22}$.

From the above observations on the cavity profile as well as the capillary wave propagation, the viscous damping of the capillary waves as well as the wavelength of the selected dominant wave is increased with the oil viscosity and layer thickness. Combining our argument on oil spreading at the cavity surface, we expect that the viscous attenuation and selection of the capillary waves are modified by the presence of a more viscous oil film on the bubble cavity surface, and the viscous damping rate increases with oil viscosity and layer thickness. Next, we will explore how to connect such damping on the jet ejection with the oil spreading.
Viscous damping with oil spreading. While Bo compares the gravity and capillary effects, Oh compares the viscous damping rate of the cavity collapse, $t_{\mathrm{d}}^{-1}$, and the inverse of the time of cavity collapse, $t_{c}^{-125,35}$. Our experimental observation shows that the capillary waves are progressively damped with the increase of the oil viscosity and layer thickness, indicating a higher viscous damping rate of cavity collapse. Here we derive the viscous damping rate with an oil-covered interface combining the oil spreading dynamics.

The thin oil film spreading along the cavity surface can be considered as a viscous surface film with two-dimensional shear viscosity $\mu_{\mathrm{o}} h_{\mathrm{e}}$ and dilatational viscosity $3 \mu_{\mathrm{o}} h_{\mathrm{e}}{ }^{38}$, where $h_{\mathrm{e}}$ is the film thickness (see Fig. 6d and 'Methods' for the detailed derivation). Thus, for such a compound interface, we obtain a leading order approximation for the total dissipation rate of a capillary wave with a wavelength $\lambda$ and wave number $k \sim \lambda^{-1}$ as

$$
\langle D\rangle=\frac{4\left(\mu_{\mathrm{w}}+\mu_{\mathrm{o}} k h_{\mathrm{e}}\right) k^{2}}{\rho_{\mathrm{w}}} E_{0} .
$$

Since the amplitude of capillary waves, $\alpha$ decreases exponentially in time by $\mathrm{e}^{-t / t_{\mathrm{d} \lambda} 39}$, while $E_{0} \propto \alpha^{2} \propto \mathrm{e}^{-2 t / t_{\mathrm{d} \lambda}}$ with $t_{\mathrm{d} \lambda}^{-1}$ as the effective damping rate of the capillary wave with a wavelength $\lambda$, we consider

$$
\frac{\mathrm{d} E_{0}}{\mathrm{~d} t}=-\langle D\rangle \text {. }
$$

Therefore, we obtain

$$
t_{\mathrm{d} \lambda}^{-1}=2 \frac{\mu_{\mathrm{w}}+\mu_{\mathrm{o}} k h_{\mathrm{e}}}{\rho_{\mathrm{w}}} k^{2} .
$$

We note that Eq. (7) also agrees with the expression for the capillary wave damping rate due to a viscous surface fluid layer by Jenkins and Jacobs ${ }^{36}$. Though there is a series of capillary waves with different $\lambda$ propagating along the cavity surface, the only characteristic length of the initial condition of the system, $R$, is adopted in $t_{\mathrm{d}}^{-1}$ which characterizes the viscous damping of the cavity collapse $25,35,40$. Following this argument, by using $k \sim R^{-1}$ in our experiment, we further obtain

$$
t_{\mathrm{d}}^{-1} \sim \frac{\mu_{\mathrm{w}}+\frac{h_{\mathrm{e}}}{R} \mu_{\mathrm{o}}}{\rho_{\mathrm{w}} R^{2}} .
$$

We observe that the oil film spreading along the cavity surface has a non-uniform thickness profile (Fig. 3b-d). For the sake of simplicity to reveal the driving physical mechanism, we approximate the average film thickness $h_{\mathrm{e}}$ with respect to the effective damping rate, which is assumed to be mainly contributed by the spreading of the macroscopic oil film considering the much smaller thickness of the precursor film in comparison. For an oil layer surrounding the bubble cavity, we consider the layer as an infinite reservoir localized at the cavity edge, which provides a flow rate $Q$ to form the oil film on the bubble cavity. $Q$ is estimated as $Q \sim 2 \pi R h_{\mathrm{i}} \bar{v}$, where $h_{\mathrm{i}}$ is the initial oil layer thickness at the boundary of the bubble cavity before spreading (Fig. 6b), and $\bar{v}$ is the average inward spreading velocity of the macroscopic oil edge. Similar to the scenario for inward spreading of a healing capillary film ${ }^{41}$, the time evolution of $h_{\mathrm{i}}$ scales with the initial oil layer thickness $h$, while $\bar{v}$ is estimated by $\gamma_{\mathrm{e}} / \mu_{\mathrm{o}}{ }^{42,43}$. Therefore, $Q \sim 2 \pi R h \gamma_{\mathrm{e}} / \mu_{\mathrm{o}}$, and the average oil thickness $h_{\mathrm{e}} \sim Q t_{\mathrm{c}} / \pi R^{2}$, where $t_{\mathrm{c}}$ is the time of cavity collapse as the capillary time (Eq. (3)). Now by scaling analysis, we obtain

$$
h_{\mathrm{e}} \sim \mathrm{Oh}_{\mathrm{o}}^{-1} h,
$$

where $\mathrm{Oh}_{\mathrm{o}}=\mu_{\mathrm{o}} / \sqrt{\rho_{\mathrm{o}} \gamma_{\mathrm{e}} R}$, showing that the oil film on the bubble cavity formed by inward spreading can be related to the initial oil layer thickness $h$ by $\mathrm{Oh}_{\mathrm{o}}$. Given the simplified 

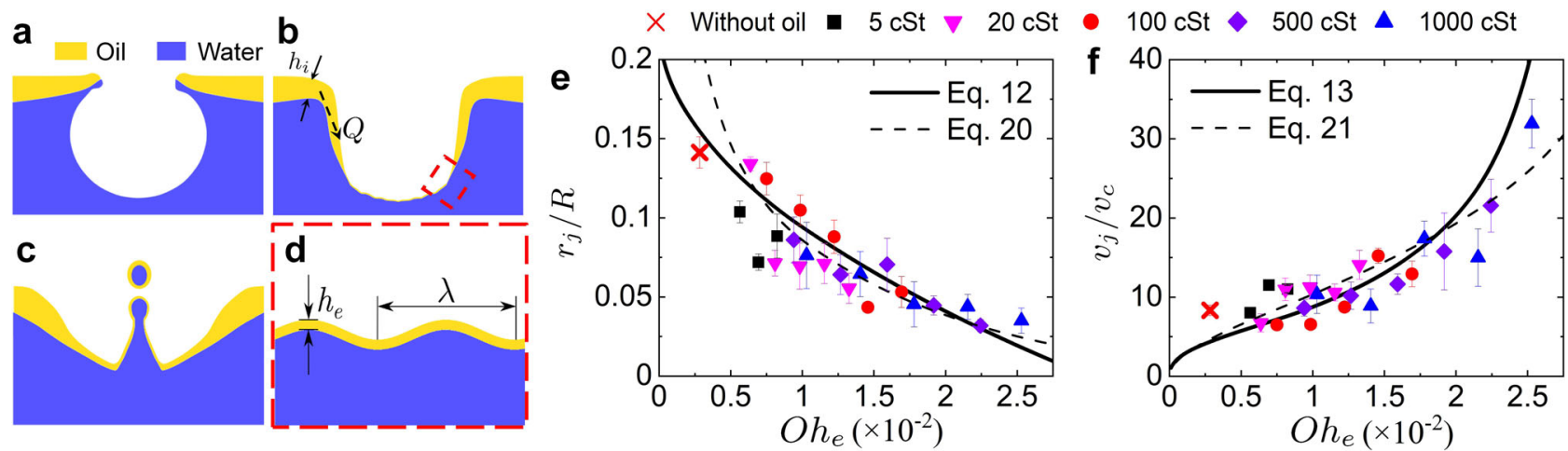

Fig. 6 Schematics of bubble bursting at an air-oil-water interface and scaling laws of jet tip radius and velocity. a Cap rupture, $\mathbf{b}$ cavity collapse, and $\mathbf{c}$ jet formation of bubble bursting at an oil-covered aqueous surface. The oil spreading along the cavity surface has a flow rate of $Q$ from the oil layer around the cavity with an initial thickness of $h_{\mathrm{i}}$. $\mathbf{d}$ Zoom-in view of the red square zone in (b), showing a capillary wave of wavelength $\lambda$ at a water surface covered with an oil film of thickness $h_{\mathrm{e}}$. Dimensionless (e) radius $r_{\mathrm{j}} / R$ and $(\mathbf{f})$ velocity $v_{\mathrm{j}} / v_{\mathrm{c}}$ of the jet tip as a function of the revised Ohnesorge number Ohe. In (e) and (f), the solid lines represent the scaling laws of Eqs. (12) and (13), and the dashed lines represent the scaling laws of Eqs. (20) and (21), respectively. Error bars are calculated as the standard deviations of data of at least 10 runs.

configuration (Fig. 6b, d) in this study, we revise Eq. (9) as $h_{\mathrm{e}} \sim a \mathrm{Oh}_{\mathrm{o}}^{b} h$, with $a$ and $b$ as the numerical factors obtained from experiments. Thus, the effective viscous damping rate of the cavity collapse is written as

$$
t_{\mathrm{d}}^{-1} \sim \frac{\mu_{\mathrm{w}}+a \mathrm{Oh}_{\mathrm{o}}^{b} \frac{h}{R} \mu_{\mathrm{o}}}{\rho_{\mathrm{w}} R^{2}}
$$

Scaling laws for the oily jetting. By modeling the dynamics of both bubble cavity collapse and oil spreading, we now propose scaling laws to predict $r_{\mathrm{j}}$ and $v_{\mathrm{j}}$ for the compound jetting. We note that $\mathrm{Bo} \approx 0.4$ was kept the same throughout all the experiments (Table 1). Meanwhile, the $\mathrm{Oh}$ can be revised adopting the effective viscous damping rate of the cavity collapse $t_{\mathrm{d}}^{-1}$ (Eq. (10)) for our experiments, yielding a revised Ohnesorge number as

$$
\mathrm{Oh}_{\mathrm{e}}=\frac{t_{\mathrm{d}}^{-1}}{t_{\mathrm{c}}^{-1}}=\frac{\mu_{\mathrm{w}}+a \mathrm{Oh}_{\mathrm{o}}^{b} \frac{h}{R} \mu_{\mathrm{o}}}{\left(\rho_{\mathrm{w}} \gamma_{\mathrm{e}} R\right)^{1 / 2}}
$$

For bubble bursting at a clean liquid surface, $r_{j}$ decreases and $v_{j}$ increases monotonically with $\mathrm{Oh}$ when $\mathrm{Oh}<\mathrm{Oh}_{\mathrm{c}}{ }^{22,25,40}$, where $\mathrm{Oh}_{\mathrm{c}} \approx 0.0305^{37}$. Our experimental results show the same trends which means $\mathrm{Oh}_{e}<\mathrm{Oh}_{c}$ (Fig. 2e, f). By balancing the viscous damping rate of the capillary wave $t_{\mathrm{d} \lambda}^{-1}$ calculated using Eq. (7) with $t_{\mathrm{c}}^{-1}$, the wavelength of the selected dominant wave can be obtained as $\lambda_{\mathrm{d}} / R \propto \mathrm{Oh}_{\mathrm{e}}^{1 / 2}$, similar to the argument for bubble bursting at a clean liquid surface with $\mathrm{Oh}<\mathrm{Oh}_{\mathrm{c}}{ }^{25}$. It was suggested that $\lambda_{\mathrm{d}}$ sets the radius at the base of the truncated coneshaped cavity at the onset of jet formation, which further determines the radius of the jet tip, yielding $r_{\mathrm{j}} / R=c_{r}(1-$ $\left.\left(\mathrm{Oh} / \mathrm{Oh}_{\mathrm{c}}\right)^{1 / 2}\right)$ with $\mathrm{Oh}<\mathrm{Oh}_{\mathrm{c}}{ }^{37}$. Therefore, with the revised Ohnesorge number $\mathrm{Oh}_{e}$, we propose that

$$
\frac{r_{\mathrm{j}}}{R}=c_{r}\left(1-\left(\frac{\mathrm{Oh}_{\mathrm{e}}}{\mathrm{Oh}_{\mathrm{c}}}\right)^{1 / 2}\right), \text { for } \mathrm{Oh}_{\mathrm{e}}<\mathrm{Oh}_{\mathrm{c}},
$$

where $c_{r}=0.2215$ as predicted by the prior work ${ }^{37}$. For bubble bursting at a clean liquid surface, prior work also argued that $v_{\mathrm{j}}$ can be calculated as the flow generated by a continuous line of sinks extending along the axis of symmetry a distance proportional to $\lambda_{\mathrm{d}}{ }^{37}$. Based on this argument, we further propose that

$$
\frac{v_{\mathrm{j}}}{v_{\mathrm{c}}}=\frac{c_{v}}{\left(-\ln \left(r_{\mathrm{j}} / R\right)\right)^{1 / 2}}\left(\frac{1}{r_{\mathrm{j}} / R}-\frac{1}{r_{\mathrm{j}} / R+4.67 \mathrm{Oh}_{\mathrm{e}}^{1 / 2}}\right), \text { for } \mathrm{Oh}_{\mathrm{e}}<\mathrm{Oh}_{\mathrm{c}},
$$

where $r_{j} / R$ is calculated by Eq. (12), and $c_{v}$ is found to be $\approx 1.5$. The smaller $c_{v}$ compared to the literature value of $4.2^{37}$ may be attributed to the deceleration of the jet tip when it rises from the bubble nadir to the surface ${ }^{37}$ and the effect of relatively large $\mathrm{Bo}^{40}$ in the current experiments.

For a wide range of experimental conditions, including viscosity of 5-1000 cSt and layer thickness of $0.67-2 \mathrm{~mm}$, we find that Eqs. (12) and (13) agree well with our experimental results as shown in Fig. $6 e$, f. Here, $\mathrm{Oh}_{\mathrm{e}}$ takes into account the additional viscous dissipation by the oil layer film on the cavity surface formed by spontaneous spreading, related to the oil viscosity and initial layer thickness. We note that $a=0.015$ and $b=-0.8$ in Eq. (11) obtained by experimental fitting, indicating that the average oil-film thickness $h_{\mathrm{e}}=O\left(1-10^{2} \mu \mathrm{m}\right) \ll R$, are consistent with the experimental observations and the assumption for the oil film to be considered as a thin surface film. In addition, the fitting value of $b$ is close to -1 obtained by the scaling analysis for Eq. (9). Meanwhile, we found that using the same $a$ and $b$, the $\mathrm{Oh}_{\mathrm{e}}$ can also be adopted in the scaling laws proposed by other studies to fairly describe our experimental results ${ }^{29,35}$ (see Fig. 6e, f and 'Methods' for the detailed information). Overall, the proposed revised Ohnesorge number and scaling laws universally capture the jet dynamics of bubble bursting at an oil-covered aqueous surface in the current experimental range.

\section{Discussion}

Our findings demonstrate a distinct transport mechanism related to dynamics of the bubble bursting jetting at a compound interface. Although previous studies have reported evidence of oily aerosol generation by bubble bursting at a contaminated surface $^{44,45}$, only film droplets are considered to be responsible for the dispersion of the insoluble surface contaminants, including bacteria and aliphatic-rich hydrophobic organic species in the surface microlayer ${ }^{15,19}$, while the jet droplets are believed to only contribute to the transport of more soluble organic species from the underlying seawater ${ }^{15,46,47}$. In this study, we highlight the 
formation of a compound oily jet, resulting from the interplay of interfacial physicochemistry and bubble-bursting hydrodynamics. In a complete-wetting state, even surface contaminants can be dispersed by the bubble bursting jet due to the spontaneous spreading. In addition, the associated jetting dynamics will be affected by such a structurally complex interface, from the change of the effective interfacial tension as well as the viscous damping. By measuring the jet droplet compositions, our study directly evidences marine oily aerosol formation by bubble bursting jets with oil slicks, and may also serve as a valuable step to further consider the role of physicochemistry in the hydrodynamic phenomena related to bubbles. Meanwhile, although the jet radius is within $50-200 \mu \mathrm{m}$ due to the large bubble radius in the current experiments, our scaling laws predict that the oily jet droplet size can be $O(1-10 \mu \mathrm{m})$ with smaller bubble radius $R=O\left(10^{2}-10^{3}\right.$ $\mu \mathrm{m})$. Therefore, the compound jet droplets can potentially remain airborne for a significant amount of time, contributing to net transport of species from the bulk liquid to the atmosphere.

The results we report suggest potential environmental, biological, and public health consequences for bubble bursting in nature and industrial processes. For instance, the interface separating the ocean from Earth's atmosphere is covered by the sea-surface microlayer, which is a physicochemically complex organic film containing significant amounts of lipids, proteins, and hydrocarbons ${ }^{48,49}$. The sea-surface microlayer is structurally similar to our compound interface, and our work suggests that bubble bursting can transfer the organic materials in the seasurface microlayer by the compound jetting. With respect to the airborne disease and pollutant transmission ${ }^{10,45}$, our work demonstrates that the physicochemistry of the interface, such as the wettability, is required to be taken into consideration to investigate the aerosol size and droplet engulfment of the organic matter. It has been found that bacterial growth at the oil-water interface alters the wettability by secretion of biosurfactants $\mathrm{s}^{50}$, and thus our work may shed some light on the role of the microbial-enriched, structurally complex interface on droplet erupting from bursting bubbles as well as the subsequent airborne transmission modeling. Meanwhile, bursting bubbles are considered to produce sufficient mechanical stresses to be biologically relevant to organisms living in the sea-surface microlayer, including cell damage and vegetative reproduction at the microscale $9,51,52$. Our work suggests that the presence of an insoluble viscous layer may alter the mechanical stress and energy transport during jet formation, thus facilitating smaller jet droplet production. In addition, prior work has shown that the seasurface microlayer may even behave as a viscoelastic layer ${ }^{48,53,54}$, showing more complicated rheological response than the oil used in the current work. The rheological effect of the oil layer will be further considered in our future work.

In conclusion, we report that bubble bursting can disperse highly viscous surface organic matter as compound jet droplets into air, and unravel the physical mechanism related to the jetting dynamics at an oil-covered aqueous surface. We believe that our findings advance our insights into the interfacial dynamics of complex fluids, as well as the size evaluation for oily aerosol generated by bursting bubbles regarding marine environment and public health.

\section{Methods}

Materials. We used deionized water (resistivity $=18.2 \mathrm{M} \Omega \cdot \mathrm{cm}$, Smart2Pure 3 UV/UF, Thermo Fisher Scientific) as the bulk liquid, and silicone oils (SigmaAldrich; viscosity $=5,20,100,500$, and $1000 \mathrm{cSt}$ at $25^{\circ} \mathrm{C}$ and $1 \mathrm{~atm}$ ) as the oil layer. Their surface tensions $\gamma_{\text {oa }}$ (or $\gamma_{\text {wa }}$ ) and interfacial tension $\gamma_{\text {ow }}$ were measured using the pendant-drop method, and their density $\rho$ and viscosity $\mu$ are listed in Table 1.
High-speed imaging. A square transparent acrylic container with a cross-section area of $120 \times 120 \mathrm{~mm}^{2}$ and a height of $40 \mathrm{~mm}$ was designed to hold the liquids (Fig. 1a). A stainless-steel needle (18 Gauge) was used to generate the gas bubble by a syringe pump (11 Pico Plus Elite, Harvard Apparatus). The distance between the water surface and the needle was set as $5 \mathrm{~mm}$, and an oil layer with a thickness of $h$ was deposited onto the water surface using a pipette. The cavity collapse and jet formation were recorded synchronously from the side view by two high-speed cameras (FASTCAM Mini AX200, Photron) mounted with focus lenses using a magnification of 1.8-2.3, illuminated by two LED panels, respectively. We used a frame rate of 10,000 frames per second, an exposure time of 20-50 $\mu$ s, and an image resolution of $1024 \times 762$ pixels. The obtained images were further processed using Fiji (ImageJ) $)^{55}$.

Viscous damping of the capillary waves at an oil-covered aqueous surface. We consider the damping rate of the surface capillary waves in a system that consists of a thin layer of oil with a finite thickness, $h_{\mathrm{e}}$, floating on water with an infinite depth, while the bubble cavity curvature is neglected (Fig. 6d). For a capillary wave with a wave number $k$, the boundary layer thickness in the oil layer is $^{38}$

$$
h_{\mathrm{bo}}=\sqrt{\frac{2 \mu_{\mathrm{o}}}{\omega \rho_{\mathrm{o}}}},
$$

where the capillary frequency

$$
\omega=\sqrt{\frac{\gamma_{\mathrm{e}} k^{3}}{\rho_{\mathrm{w}}}} .
$$

Regarding the dominant capillary wave with $k \sim R^{-1}$ in our bubble bursting experiments, $h_{\mathrm{bo}}$ is calculated to $O(1 \mathrm{~mm})$, while the observed thickness of the oil layer on the bubble cavity surface is much smaller than the bubble radius. Given that $h_{\mathrm{bo}} \gg h_{\mathrm{e}}$, the oil layer can be considered as a viscous surface film, with twodimensional shear viscosity $\eta_{1}=\mu_{\mathrm{o}} h_{\mathrm{e}}$ and two-dimensional dilatational viscosity $\eta_{2}=3 \mu_{\mathrm{o}} h_{\mathrm{e}}^{38}$. Additionally, we estimate boundary layer thickness in water as ${ }^{56}$

$$
h_{\mathrm{bw}}=\sqrt{\frac{2 \mu_{\mathrm{w}}}{\omega \rho_{\mathrm{w}}}}
$$

and we obtain $k h_{\mathrm{bw}} \ll 1$ and $k h_{\mathrm{w}} \gg 1$, where $h_{\mathrm{w}}$ is the water depth in the experiments. Therefore, we can adopt a deep water assumption. From the previous studies of a thin floating fluid layer ${ }^{38,56}$, the total dissipation rate of the capillary waves is written as

$$
\langle D\rangle=\frac{4 \mu_{\mathrm{w}} k^{2}}{\rho_{\mathrm{w}}} \frac{2+\zeta}{2+2 \zeta+\zeta^{2}} E_{0}+\left(\frac{1}{2} \frac{\mu_{\mathrm{w}} \omega k^{2}}{\rho_{\mathrm{w}}}\right)^{\frac{1}{2}} \frac{2 \zeta+\zeta^{2}}{2+2 \zeta+\zeta^{2}} E_{0} .
$$

Here \langle\rangle implies an average over one cycle of the oscillation, $E_{0}$ is the total energy of wave oscillation, and $\zeta$ is a dimensionless number comparing the effect of surface viscosity and the bulk water viscosity as

$$
\zeta=\left(\frac{1}{2} \mu_{\mathrm{w}} \rho_{\mathrm{w}} \omega\right)^{-\frac{1}{2}} k^{2}\left(\eta_{1}+\eta_{2}\right)=4\left(\frac{1}{2} \mu_{\mathrm{w}} \rho_{\mathrm{w}} \omega\right)^{-\frac{1}{2}} k^{2} \mu_{\mathrm{o}} h_{\mathrm{e}} .
$$

The first term on the right-hand side of Eq. (17) is the dissipation rate from the irrotational motion and the interaction between the irrotational motion and the water boundary layer motion, while the second term is the dissipation rate from the rotational motion and shear flow in the surface layer. In our experiments, $\zeta \ll 1$. Thus, we obtain a leading order approximation for the total dissipation rate as

$$
\langle D\rangle=\frac{4\left(\mu_{\mathrm{w}}+\mu_{\mathrm{o}} k h_{\mathrm{e}}\right) k^{2}}{\rho_{\mathrm{w}}} E_{0} .
$$

Alternative scaling laws for the oily jetting. Considering the energy balance during cavity collapse, the surface energy of the bubble collapse, minus the viscous dissipation associated to the motion induced by the capillary wave, provides the surplus of the mechanical energy forming the jet ${ }^{27,35}$. Using a balance of the inertia, capillarity and viscosity effects at the onset of the jet ejection, the following scaling laws using Oh have been obtained as ${ }^{35,40} r_{\mathrm{j}} / R=k_{d}(0.043-\mathrm{Oh})^{5 / 4} \mathrm{Oh}^{-1 / 2}$ and $v_{\mathrm{j}} / v_{\mathrm{c}}=k_{v}(1+2.2 \mathrm{Bo})^{-3 / 4}(0.043-\mathrm{Oh})^{-3 / 4} \mathrm{Oh}^{1 / 2}$, where $k_{d}$ and $k_{v}$ are prefactors depending on the measurement position with $\mathrm{Bo}<1$ and $\mathrm{Oh}<\mathrm{Oh}_{\mathrm{c}}{ }^{27,29}$. Therefore, with the revised Ohnesorge number $\mathrm{Oh}_{\mathrm{e}}<\mathrm{Oh}_{\mathrm{c}}$, we can obtain

$$
\begin{gathered}
\frac{r_{j}}{R}=k_{d}\left(0.043-\mathrm{Oh}_{\mathrm{e}}\right)^{5 / 4} \mathrm{Oh}_{\mathrm{e}}^{-1 / 2}, \\
\frac{v_{\mathrm{j}}}{v_{\mathrm{c}}}=k_{v}(1+2.2 \mathrm{Bo})^{-3 / 4}\left(0.043-\mathrm{Oh}_{\mathrm{e}}\right)^{-3 / 4} \mathrm{Oh}_{\mathrm{e}}^{1 / 2} .
\end{gathered}
$$

Figure 6e, f shows that Eqs. (20) and (21) can also capture our experiment results well, with $k_{d}=0.6$ and $k_{v}=12.6$, which are similar with the literature values for the moment where the jet tip reaches the undisturbed water level ${ }^{35,40}$. This verifies that the revised Ohnesorge number $\mathrm{Oh}_{\mathrm{e}}$ we proposed can be adopted as the dominant 
dimensionless number characterizing the bursting dynamics of gas bubbles at an oil-covered aqueous surface.

\section{Data availability}

The data that support the findings of this study are available from the corresponding author upon reasonable request.

Received: 18 March 2021; Accepted: 17 September 2021;

Published online: 02 November 2021

\section{References}

1. Blanchard, D. \& Woodcock, A. Bubble formation and modification in the sea and its meteorological significance. Tellus 9, 145-158 (1957).

2. MacIntyre, F. Flow patterns in breaking bubbles. J. Geophys. Res. 77, 5211-5228 (1972).

3. Ghabache, E., Antkowiak, A., Josserand, C. \& Séon, T. On the physics of fizziness: how bubble bursting controls droplets ejection. Phys. Fluids 26, 121701 (2014)

4. Lewis, E. R., Lewis, E. R., Lewis, R., Karlstrom, K. E. \& Schwartz, S. E. Sea Salt Aerosol Production: Mechanisms, Methods, Measurements, and Models. Vol. 152 (American Geophysical Union, 2004).

5. Veron, F. Ocean spray. Annu. Rev. Fluid Mech. 47, 507-538 (2015).

6. Blanchard, D. C. The electrification of the atmosphere by particles from bubbles in the sea. Prog. Oceanogr. 1, 73-202 (1963).

7. Spiel, D. E. On the births of jet drops from bubbles bursting on water surfaces. J. Geophys. Res. 100, 4995-5006 (1995).

8. Lhuissier, H. \& Villermaux, E. Bursting bubble aerosols. J. Fluid Mech. 696, 5 (2012).

9. Hariadi, R. F., Winfree, E. \& Yurke, B. Determining hydrodynamic forces in bursting bubbles using dna nanotube mechanics. Proc. Natl Acad. Sci. USA 112, E6086-E6095 (2015).

10. Bourouiba, L. The fluid dynamics of disease transmission. Annu. Rev. Fluid Mech. 53, 473-508 (2021).

11. Murphy, D. et al. An in-depth survey of the oil spill literature since 1968: long term trends and changes since deepwater horizon. Mar. Pollut. Bull. 113 371-379 (2016).

12. Blanchard, D. C. The ejection of drops from the sea and their enrichment with bacteria and other materials: a review. Estuaries 12, 127-137 (1989).

13. Weber, M. E., Blanchard, D. C. \& Syzdek, L. D. The mechanism of scavenging of waterborne bacteria by a rising bubble. Limnol. Oceanogr. 28, 101-105 (1983).

14. Su, L., Xu, Z. \& Masliyah, J. Role of oily bubbles in enhancing bitumen flotation. Miner. Eng. 19, 641-650 (2006).

15. Wang, X. et al. The role of jet and film drops in controlling the mixing state of submicron sea spray aerosol particles. Proc. Natl Acad. Sci. USA 114, 6978-6983 (2017).

16. Feng, J. et al. Nanoemulsions obtained via bubble-bursting at a compound interface. Nat. Phys 10, 606-612 (2014).

17. Feng, J. et al. A scalable platform for functional nanomaterials via bubblebursting. Adv. Mater. (Weinheim, Ger.) 28, 4047-4052 (2016).

18. Walls, P. L. \& Bird, J. C. Enriching particles on a bubble through drainage: measuring and modeling the concentration of microbial particles in a bubble film at rupture. Elementa 5, 34 (2017).

19. Poulain, S. \& Bourouiba, L. Biosurfactants change the thinning of contaminated bubbles at bacteria-laden water interfaces. Phys. Rev. Lett. 121, 204502 (2018).

20. Sanjay, V., Lohse, D. \& Jalaal, M. Bursting bubble in a viscoplastic medium. J. Fluid Mech. 922, A2 (2021).

21. Krishnan, S., Hopfinger, E. J. \& Puthenveettil, B. A. On the scaling of jetting from bubble collapse at a liquid surface. J. Fluid Mech. 822, 791 (2017).

22. Brasz, C. F. et al. Minimum size for the top jet drop from a bursting bubble. Phys. Rev. Fluids 3, 074001 (2018).

23. Eggers, J. Nonlinear dynamics and breakup of free-surface flows. Rev. Mod. Phys. 69, 865 (1997).

24. Chen, A. U., Notz, P. K. \& Basaran, O. A. Computational and experimental analysis of pinch-off and scaling. Phys. Rev. Lett. 88, 174501 (2002).

25. Gordillo, J. \& Rodríguez-Rodríguez, J. Capillary waves control the ejection of bubble bursting jets. J. Fluid Mech. 867, 556-571 (2019).

26. Blanco-Rodríguez, F. J. \& Gordillo, J. On the sea spray aerosol originated from bubble bursting jets. J. Fluid Mech. 886 R2 (2020).

27. Gañán-Calvo, A. M. Scaling laws of top jet drop size and speed from bubble bursting including gravity and inviscid limit. Phys. Rev. Fluids 3, 091601 (2018).

28. Walls, P. L., Henaux, L. \& Bird, J. C. Jet drops from bursting bubbles: how gravity and viscosity couple to inhibit droplet production. Phys. Rev. E 92, 021002 (2015).
29. Berny, A., Deike, L., Séon, T. \& Popinet, S. Role of all jet drops in mass transfer from bursting bubbles. Phys. Rev. Fluids 5, 033605 (2020)

30. Stewart, P. S., Feng, J., Kimpton, L. S., Griffiths, I. M. \& Stone, H. A. Stability of a bi-layer free film: simultaneous or individual rupture events? J. Fluid Mech. 777, 27-49 (2015).

31. Dussaud, A. D. \& Troian, S. M. Dynamics of spontaneous spreading with evaporation on a deep fluid layer. Phys. Fluids 10, 23-38 (1998)

32. Wodlei, F., Sebilleau, J., Magnaudet, J. \& Pimienta, V. Marangoni-driven flower-like patterning of an evaporating drop spreading on a liquid substrate. Nat. commun. 9, 1-12 (2018).

33. Camp, D. W. \& Berg, J. C. The spreading of oil on water in the surface-tension regime. J. Fluid Mech. 184, 445-462 (1987).

34. Fay, J. A. The Spread of Oil Slicks on a Calm Sea. 53-63 (Springer, 1969). .

35. Gañán-Calvo, A. M. Revision of bubble bursting: universal scaling laws of top jet drop size and speed. Phys. Rev. Lett. 119, 204502 (2017).

36. Jenkins, A. D. \& Jacobs, S. J. Wave damping by a thin layer of viscous fluid. Phys. Fluids 9, 1256-1264 (1997).

37. Blanco-Rodríguez, F. J. \& Gordillo, J. On the jets produced by drops impacting a deep liquid pool and by bursting bubbles. J. Fluid Mech. 916, A37 (2021).

38. Jenkins, A. D. \& Dysthe, K. B. The effective film viscosity coefficients of a thin floating fluid layer. J. Fluid Mech. 344, 335-337 (1997).

39. Lamb, H. Hydrodynamics (Dover Publications, 1945).

40. Deike, L. et al. Dynamics of jets produced by bursting bubbles. Phys. Rev. Fluids 3, 013603 (2018).

41. Zheng, Z. et al. Healing capillary films. J. Fluid Mech. 838, 404-434 (2018).

42. Eddi, A., Winkels, K. G. \& Snoeijer, J. H. Short time dynamics of viscous drop spreading. Phys. Fluids 25, 013102 (2013).

43. Rahman, M. R., Mullagura, H. N., Kattemalalawadi, B. \& Waghmare, P. R. Droplet spreading on liquid-fluid interface. Colloids Surf. A 553, 143-148 (2018).

44. Ehrenhauser, F. S. et al. Bubble bursting as an aerosol generation mechanism during an oil spill in the deep-sea environment: laboratory experimental demonstration of the transport pathway. Environ. Sci. Process. Impacts 16, 65-73 (2014).

45. Sampath, K. et al. Aerosolization of crude oil-dispersant slicks due to bubble bursting. J. Geophys. Res. 124, 5555-5578 (2019).

46. Wang, X. et al. Microbial control of sea spray aerosol composition: a tale of two blooms. ACS Cent. Sci. 1, 124-131 (2015).

47. Burrows, S. M. et al. A physically based framework for modeling the organic fractionation of sea spray aerosol from bubble film langmuir equilibria. Atmos. Chem. Phys. 14, 13601-13629 (2014).

48. Cunliffe, M. et al. Sea surface microlayers: a unified physicochemical and biological perspective of the air-ocean interface. Prog. Oceanogr. 109, 104-116 (2013).

49. Tang, D. et al. Ecological response of phytoplankton to the oil spills in the oceans. Geomat. Nat. Haz. Risk 10, 853-872 (2019).

50. Kowalewski, E., Rueslåtten, I., Steen, K., Bødtker, G. \& Torsæter, O. Microbia improved oil recovery-bacterial induced wettability and interfacial tension effects on oil production. J. Petrol. sci. Eng. 52, 275-286 (2006).

51. Cherry, R. S. \& Hulle, C. T. Cell death in the thin films of bursting bubbles. Biotechnol. Prog. 8, 11-18 (1992).

52. McRae, O., Mead, K. R. \& Bird, J. C. Aerosol agitation: quantifying the hydrodynamic stressors on particulates encapsulated in small droplets. Phys. Rev. Fluids 6, L031601 (2021).

53. Frew, N. M. \& Nelson, R. K. Scaling of marine microlayer film surface pressure-area isotherms using chemical attributes. J. Geophys. Res. 97, 5291-5300 (1992)

54. Boniewicz-Szmyt, K. \& Pogorzelski, S. J. Evolution of natural sea surface films: a new quantification formalism based on multidimensional space vector. Environ. Sci. Pollut. Res. 25, 4826-4836 (2018).

55. Schindelin, J. et al. Fiji: an open-source platform for biological-image analysis. Nat. Methods 9, 676-682 (2012).

56. Miles, J. W. Surface-wave damping in closed basins. Proc. R. Soc. London, Ser A 297, 459-475 (1967).

\section{Acknowledgements}

The authors thank Hong Liu and Dr. Leonardo Chamorro, University of Illinois at Urbana-Champaign, for their assistance with the oil concentration measurement for the oily jet droplets.

\section{Author contributions}

B.J. and J.F. conceived the initial idea for this research and designed the experiments. B.J and Z.Y. conducted the experiments and analyzed the results. Z.Y., B.J., and J.F. conducted the theoretical analysis. B.J., Z.Y., and J.F. contributed to discussing the results and writing and revising the paper.

\section{Competing interests}

The authors declare no competing interests. 


\section{Additional information}

Supplementary information The online version contains supplementary material available at https://doi.org/10.1038/s41467-021-26382-w.

Correspondence and requests for materials should be addressed to Jie Feng.

Peer review information Nature Communications thanks the anonymous reviewers for their contribution to the peer review of this work.

Reprints and permission information is available at http://www.nature.com/reprints

Publisher's note Springer Nature remains neutral with regard to jurisdictional claims in published maps and institutional affiliations. (c) (1) Open Access This article is licensed under a Creative Commons Attribution 4.0 International License, which permits use, sharing, adaptation, distribution and reproduction in any medium or format, as long as you give appropriate credit to the original author(s) and the source, provide a link to the Creative Commons license, and indicate if changes were made. The images or other third party material in this article are included in the article's Creative Commons license, unless indicated otherwise in a credit line to the material. If material is not included in the article's Creative Commons license and your intended use is not permitted by statutory regulation or exceeds the permitted use, you will need to obtain permission directly from the copyright holder. To view a copy of this license, visit http://creativecommons.org/ licenses/by/4.0/.

(C) The Author(s) 2021 